\title{
Ärzte vermeiden Behandlungen am Lebensende
}

\author{
Viele Patienten wünschen sich einen Tod zu Hause, möglichst ohne unnötig verlängertes \\ Leiden. Das sehen gerade Ärzte so, die beim Thema Sterben ja Insiderwissen haben.
}

_ Aus den Registern der Jahre 20042011 von vier US-Bundesstaaten wurden die Daten von Personen im Alter über 65 Jahren entnommen, darunter 2.396 Ärzte, 2.081 Rechtsanwälte als Kontrollgruppe mit vergleichbarem Sozial- und Bildungsstatus sowie 665.579 Personen aus der Allgemeinbevölkerung, die nicht im Gesundheitswesen tätig waren. Für die letzten fünf Jahre vor dem Tod wurden chirurgische Eingriffe, Behandlungen in Kliniken und auf Intensivstationen sowie die Behandlungskosten eruiert, außerdem der Ort des Todes (Klinik oder zu Hause).

Bei der Auswertung wurden Alter, Geschlecht, Begleiterkrankungen, Ethnie und regionale Herkunft berücksich- tigt. Im Vergleich mit der Allgemeinbevölkerung starben Ärzte seltener in einer Klinik (27,9\% vs. $32,0 \%)$, hatten weniger chirurgische Eingriffe (25,1\% vs. $27,6 \%)$ und waren seltener auf einer Intensivstation (25,8\% vs. $27,6 \%)$. Von den Rechtsanwälten starben sogar 32,7\% im Krankenhaus. Während alle genannten Unterschiede signifikant waren, war bei den Behandlungskosten nur ein Trend erkennbar.

- Weissman JS, Cooper Z, Hyder J A et al. End-of-Life Care Intensity for Physicians, Lawyers, and the General Population. JAMA. 2016;315:303-5

\section{KOMMENTAR}

Drei von fünf Behandlungsmaßnahmen am Lebensende erhielten Ärzte seltener als die
Allgemeinbevölkerung. Obwohl die Unterschiede gering sind, spricht das für eine weniger aggressive Behandlung von Ärzten. Da Ärzte aus eigener Erfahrung die Umstände am Lebensende kennen, spricht vieles für eine persönliche Mitwirkung am Lebensende. Zwischen den beiden Akademikergruppen gibt es nur einen signifikanten Unterschied: Ärzte sterben seltener im Krankenhaus als Rechtsanwälte - wohl weil sie es vermeiden wollen.

Gleichermaßen interessant und erfreulich ist, dass die Behandlungskosten zwischen den drei Gruppen nicht signifikant differieren. Tendenziell sind sie bei Ärzten mit 21.022 USDollar am geringsten (Allgemeinbevölkerung: 22.302, Rechtsanwälte: 22.839).

Prof. Dr. med. H. Holzgreve

\section{Kleienpilzflechte macht die Haut scheckig}

\begin{abstract}
Eine 24-jährige Frau hatte seit zwölf Jahren eine ausgedehnte, konfluierende Hypopigmentierung an der Außenseite der Oberarme (Abb. A). Die Hautveränderungen waren vor allem im Sommer ausgeprägt und hatten sich zwei oder drei Jahre vor der Untersuchung sogar über den gesamten Oberkörper ausgebreitet. Hinzu kam eine starke Hautschuppung vor allem nach dem Duschen. Die Frau befürchtete nun, dass sich die Effloreszenzen auf das Gesicht ausdehnen könnten. Therapieversuche mit mehreren topisch applizierten Antimykotika hatten nicht zu einer dauerhaften Remission geführt.

Zum Ausschluss einer Vitiligo wurde eine Hautbiopsie durchgeführt. Spezialfärbungen mit Melanin-A zeigten eine normale Verteilung von Melanozyten (Abb. B). Eine PAS-Färbung ließ in der oberflächlichen Epidermis Hefepilze erkennen (Abb.C). Eine Hautschuppung, die in vor allem bei Hautdehnung auftritt, spricht stark für eine Pityriasis versicolor. Für die Diagnosestellung empfiehlt sich die mikroskopische Untersuchung von abgeschabten Schuppen nach Vorbehandlung mit Kaliumhydroxid oder Chlorazol Black E. Angesichts des ausgedehnten
\end{abstract}
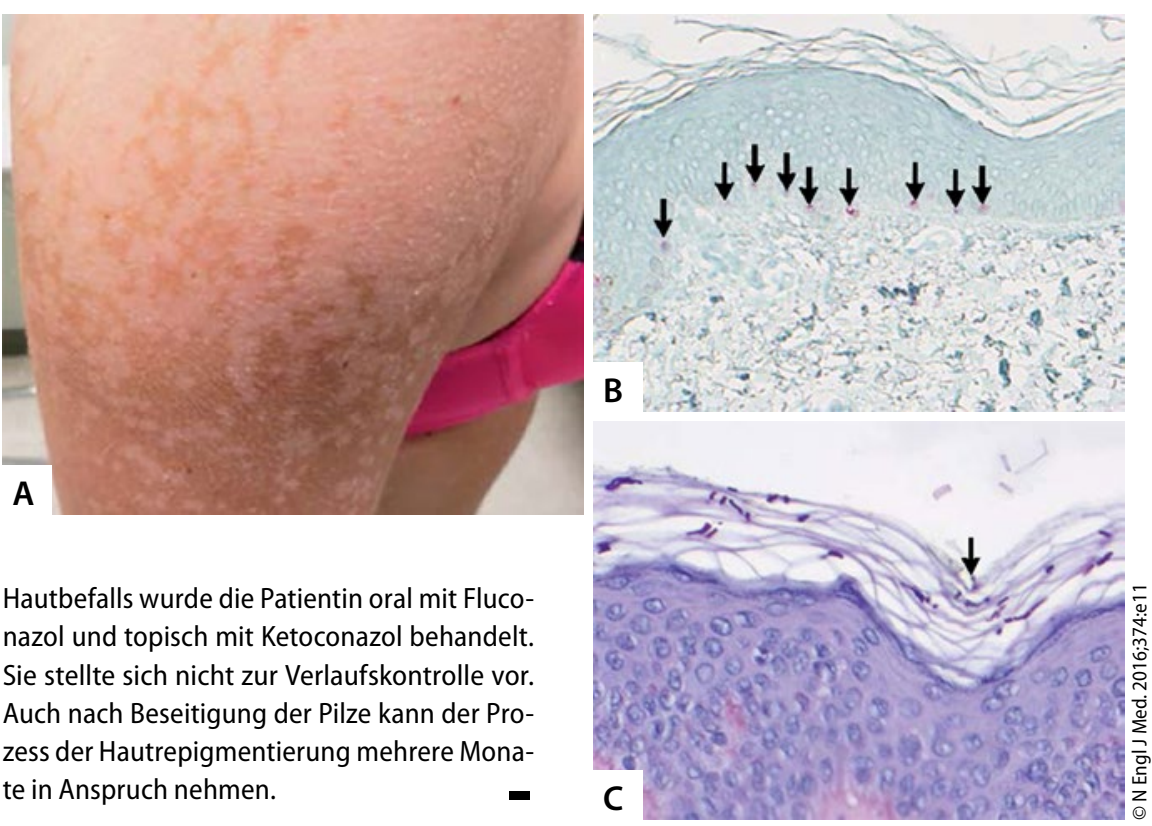

Hautbefalls wurde die Patientin oral mit Fluconazol und topisch mit Ketoconazol behandelt. Sie stellte sich nicht zur Verlaufskontrolle vor. Auch nach Beseitigung der Pilze kann der Prozess der Hautrepigmentierung mehrere Monate in Anspruch nehmen.

Prof. Dr. med. H. S. FüeßI

- Holliday A, GriderD (acholliday@gmail.com). Tinea versicolor. NEngl J Med. 2016;374:e11
Hypopigmentierung an den Oberarmen (A), normale Verteilung von Melanozyten in der Hautbiopsie (B), Pilze in der oberen Epidermis nach PAS-Färbung (C). 